Besser auf zweimal verteilen

\title{
Darmreinigung bringt die intestinale Flora nachhaltig aus der Balance
}

\begin{abstract}
Die Darmreinigung als Vorbereitung für eine Koloskopie führt kurzfristig zu einer erheblichen Veränderung der natürlichen Darmflora. Wie eine finnische Studie zeigt, erholt sich das Mikrobiom danach zwar wieder; die Art, wie man die Spüllösung dosiert, spielt dabei jedoch eine entscheidende Rolle.
\end{abstract}

\section{Schnellere Erholung des Mikrobioms,} wenn in zwei Schritten gespült wird.

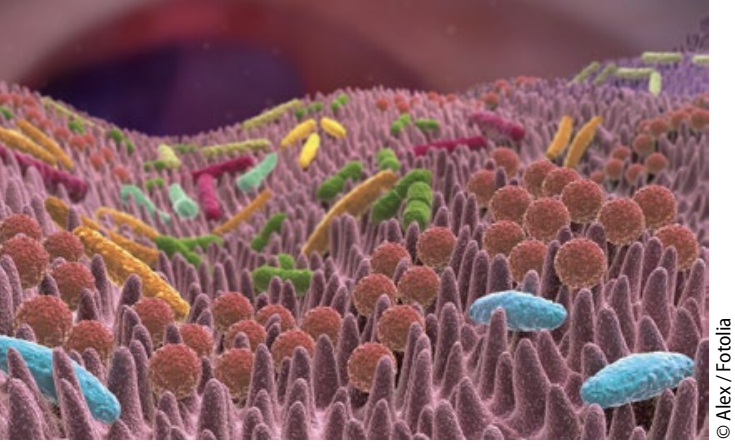

Produkte zur Darmreinigung werden routinemäßig vor Koloskopien im Rahmen der Krebsvorsorge eingesetzt. Durch osmotische Kräfte bewirken sie, dass mehr Flüssigkeit in den Darm gelangt und Darminhalt ausgespült wird - mitsamt den Bakterien, aus denen sich die natürliche Darmflora zusammensetzt. Zusätzlich gelangt durch das Spülen auch Sauerstoff in den ursprünglich anaeroben Darmtrakt. Ein finnisches Forscherteam hat nun untersucht, welche Auswirkungen das auf die Zusammensetzung des Mikrobioms im Darm hat.

Dazu wurden aus den Teilnehmern zwei Gruppen gebildet: Die erste erhielt die erforderlichen zwei Liter Spüllösung aufgeteilt auf zwei Dosen, eine am Vorabend, die zweite am Morgen der Darmspiegelung. In Gruppe 2 wurde die gesamte Dosis auf einmal, am Morgen der Untersuchung, verabreicht. Sowohl vor als auch nach der Lavage - an den Tagen 1, 14 und 28 - wurden Stuhlproben genommen und mithilfe von Gentests analysiert. Ergebnis: Kurz nach der Spülung war die Keimzahl insgesamt um den Faktor 34,7 gesunken. In Gruppe 1 (Split-dose-Gruppe) war der Abfall jedoch deutlich stärker als in Gruppe 2 (64,7- bzw. 25,3-fach).

Allerdings hatten sich einige Keime unmittelbar nach der Lavage sogar vermehrt, und zwar vor allem in Gruppe 2. Hier fanden sich nun insbesondere Proteobakterien sowie Dorea formicigenerans. Wie die Autoren betonen, findet sich ein solches Muster auch bei Patienten mit chronisch entzündlichen Darmerkrankungen, teilweise auch beim Reizdarm. „Es ist nicht auszuschließen, dass der Anstieg solcher Organismen gesundheitsschädigende Effekte haben könnte", so die Wissenschaftler.

Tatsächlich hatte die Gruppe $2 \mathrm{im}$ Mittel länger gebraucht, um sich von der Darmreinigung zu erholen. So war erst bei der Messung an Tag 28 das Mikrobiom weitgehend wiederhergestellt.

Dazu passt auch der beobachtete Anstieg der fäkalen Serinproteasen. Diese können die Durchlässigkeit der Darmwand erhöhen und möglicherweise $z u$ einer viszeralen Überempfindlichkeit beitragen.

Die Befunde sind für die Autoren ein weiteres Argument dafür, die Spüllösung auf zwei Dosen aufzuteilen, vor allem bei Menschen, die ohnehin unter Verdauungsproblemen leiden. Das Splitting-Regime wird auch von der Deutschen Gesellschaft für Verdauungs- und Stoffwechselkrankheiten (DGVS) befürwortet.

- eo

- Jalanka J et al. Effects of bowel cleansing on the intestinal microbiota. Gut 2015;64:1562-68
Antibiotika bei stabiler COPD

\section{Dauertherapie ohne Effekt auf die Keimlast}

Patienten mit stabiler COPD prophylaktisch mit Antibiotika zu behandeln, ist offenbar wenig sinnvoll: Die Therapie erhöht die Resistenzrate, ohne die Bakterienlast im Sputum signifikant zu vermindern.

In einer britischen Studie erhielten 86 mittelschwer bis schwer erkrankte Patienten mit stabiler COPD 13 Wochen lang entweder Moxifloxacin $(400 \mathrm{mg} / \mathrm{d}$ für 5 Tage alle 4 Wochen, $\mathrm{n}=21$ ), Doxycyclin $(100 \mathrm{mg} / \mathrm{d}, \mathrm{n}=22)$, Azithromycin (3 x wöchentlich $250 \mathrm{mg}, \mathrm{n}=22$ ) oder Placebo $(n=21)$. Primärer Studienendpunkt war die Veränderung der Bakterienzahlen in Sputumkulturen. Mit einer Reduktion von $62 \%$ war der Effekt mit Moxifloxacin am größten. Der Unterschied zu Placebo war allerdings ebenso wenig signifikant wie bei den zwei anderen Antibiotika. Auch mit Hilfe einer quantitativen PCR ließ sich kein signifikanter Vorteil irgendeiner Antibiotikatherapie feststellen. Entzündungsparameter, Lungenfunktion und Gesundheitszustand wurden durch die antibiotische Therapie ebenfalls nicht signifikant verbessert. $\quad-$ bs - Brill SE et al. Thorax 2015;70:930-938

\section{Zitat der Woche}

Dr. Martin Alberer, Abteilung für Infektions- und Tropenmedizin am Klinikum der LMU München, legt zu Beginn seines Vortrags auf dem Bayerischen Internistenkongress seine Interessenkonflikte offen: „Ich führe Impfstudien für die Pharmaindustrie durch, aber die haben mir bisher noch nicht so viel Geld geboten, dass ich nicht auch mal was Kritisches zum Thema Impfungen sagen könnte." 